\title{
Asynchronous Breeding and Variable Embryonic Development Period in the Threatened Northern Leopard Frog (Lithobates pipiens) in the Cypress Hills, Alberta, Canada: Conservation and Management Implications
}

\author{
Lea A. Randall ${ }^{1,3}$, Lynne D. Chalmers ${ }^{1,2}$, Axel Moehrenschlager ${ }^{1,2}$, and Anthony P. Russell ${ }^{2}$ \\ ${ }^{1}$ Centre for Conservation Research, Calgary Zoological Society, 1300 Zoo Road NE, Calgary, Alberta T2E 7V6 Canada \\ ${ }^{2}$ Department of Biological Sciences, University of Calgary, 2500 University Drive NW, Calgary, Alberta T2N 1N4 Canada \\ ${ }^{3}$ Corresponding author: lear@calgaryzoo.com
}

Randall, Lea A., Lynne D. Chalmers, Axel Moehrenschlager, and Anthony P. Russell. 2014. Asynchronous breeding and variable embryonic development period in the threatened Northern Leopard Frog (Lithobates pipiens) in the Cypress Hills, Alberta, Canada: conservation and management implications. Canadian Field-Naturalist 128(1): 50-56.

Understanding breeding phenology is critical for establishing monitoring strategies, comprehending population dynamics, and developing conservation actions for at-risk species, such as the Northern Leopard Frog (Lithobates pipiens). The timing of spawning and hatching in the Northern Leopard Frog may be highly variable depending on regional environmental conditions, which can make establishing the timing of surveys difficult. In spring 2006, eggs were laid over 30 days (24 April to 23 May) and hatching occurred over 2 weeks (14-28 May) at three neighbouring ponds in Cypress Hills, Alberta, Canada. Although spawning occurred over a month, all eggs hatched within a 2-week period, indicating variable embryo development rate. Among 26 egg masses, eggs laid later in the season developed approximately four times faster than those laid earlier, and Akaike information criterion-ranked models suggested that both Julian date and water temperature were important predictors of embryo development rate: later spawning date and warmer water were associated with faster rates. Some egg masses survived colder temperatures than previously reported for this species. Asynchronous breeding and variable development rates reveal the need to conduct multiple surveys over the breeding season, even within a small geographic area, to document reliably the presence of egg masses and identify breeding habitat. Identification of key breeding habitat is necessary to mitigate human-caused disturbances of such regionally imperiled species.

Key Words: Northern Leopard Frog; Lithobates pipiens; amphibian; breeding; egg; conservation; embryo development; spawning period; phenology; Cypress Hills; Alberta

\section{Introduction}

Breeding phenology and the time between spawning and hatching may be highly variable within and among populations of amphibians (Thumm and Mahony 2002; Ryan and Plague 2004) as a result of an assortment of exogenous and endogenous factors (Reading 1998; Oseen and Wassersug 2002; Grant et al. 2009). This variability can make timing of population surveys challenging.

Industrial development continues to increase in Alberta, particularly in association with oil and gas extraction, and may negatively affect wildlife unless proper mitigation measures can be implemented. Permits for industrial exploration and extraction often stipulate a requirement for amphibian surveys before land development if at-risk amphibians are predicted to inhabit the area (Dr. David Prescott, Species at Risk Biologist, Alberta Environment and Sustainable Resource Development, personal communication, 29 March 2012). However, for these surveys to be effective, they must be carried out at a time and in a manner appropriate for the species of interest.

Once widely distributed and abundant in North America, the Northern Leopard Frog (Lithobates pipiens) disappeared from much of the western portion of its range in the 1970s and 80s (Roberts 1981*; Leonard et al. 1999), perhaps due to such factors as disease, drought, competition by invasive species, and habitat loss and fragmentation (COSEWIC 2009*). As a result, the western boreal-prairie populations of Northern Leopard Frogs are designated of "special concern" by the Committee on the Status of Endangered Wildlife in Canada (COSEWIC 2009*) and "threatened" under Alberta's Wildlife Act (AESRD 2012*).

We investigated predictors of spawning time and embryo development rate of Northern Leopard Frogs, such as temperature and time of year, as part of a larger study of tadpole microhabitat selection and juvenile dispersal behaviour. Natural history observations of this type may be useful for improving the probability of detection by providing information regarding the timing of the breeding period and the frequency of surveys required to identify and protect important habitat.

\section{Study Area}

Our study was conducted in and near Cypress Hills Interprovincial Park $\left(49^{\circ} 39^{\prime} \mathrm{N}, 110^{\circ} 01^{\prime} \mathrm{W}\right)$ which straddles the border between Alberta and Saskatchewan and is just north of the Sweetgrass Hills of Montana (Figure 1). The Alberta portion of the Cypress Hills is char- 


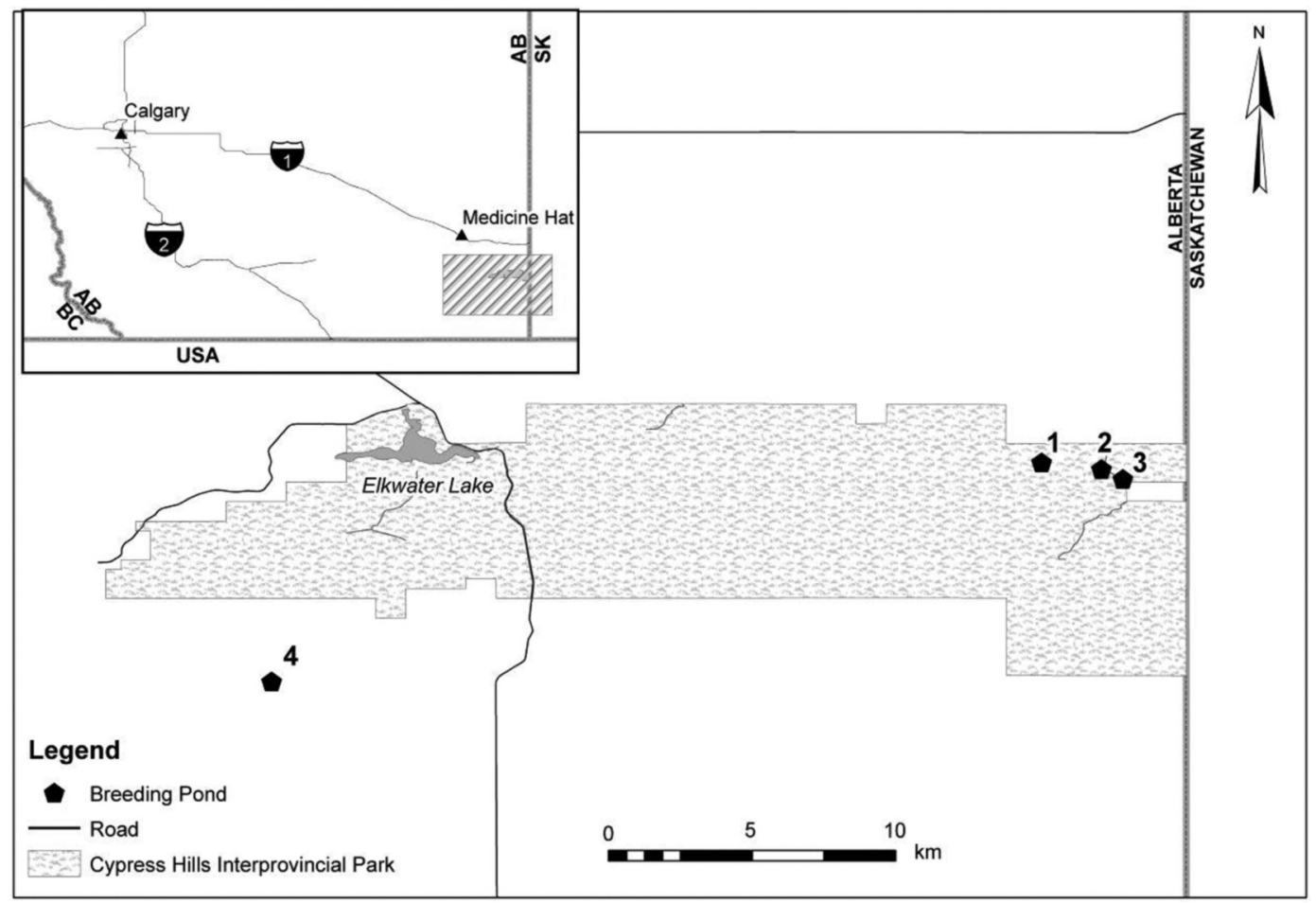

FIGURE 1. Locations of four Northern Leopard Frog (Lithobates pipiens) breeding ponds surveyed in 2006 in and near Cypress Hills Interprovincial Park, Alberta, Canada $\left(49^{\circ} 39^{\prime} \mathrm{N}, 110^{\circ} 01^{\prime} \mathrm{W}\right)$.

acterized by grasslands and boreal forest. Elevation ranges from $1370 \mathrm{~m}$ to $1465 \mathrm{~m}$, and average annual temperature is lower than that of the surrounding grassland plains (Greenlee 1981*). These environmental conditions likely present challenges to the reproductive success of Northern Leopard Frogs.

In 2006, we drove along roads and flew over the study area in a fixed-wing aircraft to identify potential Northern Leopard Frog breeding ponds within a $25-\mathrm{km}$ radius of our main study pond (Pond 1, Figure 1). Although other breeding ponds may have been present, these four represented all known breeding ponds at the time.

Pond 1 was 0.1 ha in surface area and was surrounded by Populus spp. woodlands on the east, west, and south sides; the north side had a relatively steeper slope of mixed grass prairie (Fraser 2007). Ponds 2 and 3 had surface areas of 0.17 ha and 0.04 ha, respectively, and were also surrounded by mixed grass prairie and aspen woodland. Pond 4 had a surface area of 0.24 ha and was surrounded by mixed forest, mainly white spruce (Picea glauca) on the west and north sides and deadfall on the south and east sides.

\section{Methods}

Study organism

Adult Northern Leopard Frogs are medium-sized frogs, 5-10 cm long from snout to vent (Hine et al.
1981*; Russell and Bauer 2000). Within a population, breeding period ranges from a few days to a few weeks (Wells 1977). In Alberta, spawning occurs over a short interval between late April and early June (Russell and Bauer 2000; Kendell 2002) at temperatures of $10-25^{\circ} \mathrm{C}$, although spawning may be prolonged if the temperature drops below this range (Hine et al. 1981*; Gilbert et al. 1994; Kendell 2002*). Females deposit 600-7000 eggs in a single egg mass, which they attach to submerged vegetation. Preferred water bodies are ephemeral or fishless permanent ponds or slow-moving backwaters of streams and rivers in shallow water (AESRD $2012^{*}$ ). The period from spawning to hatching may last from 5 days to 3 weeks (Russell and Bauer 2000; Werner et al. 2004) and metamorphosis typically occurs between July and August in Alberta (Kendell 2002*).

\section{Observation techniques}

Beginning approximately 30 minutes after sunset, we listened for breeding calls of adult male Northern Leopard Frogs for up to 20 minutes (AESRD 2013*). If calls were detected, we returned the following day to confirm spawning.

We conducted call and shoreline surveys from 25 April to 8 June 2006 and searched the shoreline of each pond at least once every 2 days for new egg masses to determine the duration of the breeding season and the embryo development period (Merrell 1977*; Dorcas et 
al. 2010; Paton and Harris 2010). Individual egg masses were identified by differences in either their size or shape or the stage of development of the embryos (Merrell $\left.1977^{*}\right)$. Their location along the shoreline was marked with a flag to prevent counting an egg mass twice (Gilbert et al. 1994; Dougherty et al. 2005). During each survey, egg masses were identified as hatched or unhatched (egg mass intact). Hatching was confirmed when newly hatched tadpoles were seen aggregating around the egg mass, feeding on the remaining jelly.

For each egg mass, we measured its depth below the water surface, distance to adjacent masses, and distance from the shoreline using a metre stick. During shoreline surveys, we measured the temperature of the water within $10 \mathrm{~cm}$ of each egg mass using a Hanna $\mathrm{pH}$ pen thermometer. We considered the breeding season complete once male calling had ceased and no new egg masses had been observed for at least 2 weeks.

\section{Statistical analysis}

We ran a one-way ANOVA to determine whether ponds differed with respect to water temperature when egg masses were laid, followed by Tukey's honest significant difference (HSD) test. We assessed whether residuals were normally distributed using a ShapiroWilk goodness-of-fit test ( $W>0.93)$. We used Julian date (JD) in our models to account for seasonal vari- ation of environmental factors, such as photoperiod.

To evaluate predictive factors related to embryo development, we first performed an exploratory regression analysis of embryo development rate versus JD and water temperature (WT). To further investigate the relation of JD and WT to embryo development rate we used restricted maximum likelihood mixed-model regression (JMP, version 7, SAS Institute Inc., Cary, North Carolina, USA). Pond was a random factor; all other factors were fixed. We formulated a set of candidate models that all included pond alone, pond with JD or WT, or pond with both JD and WT in an additive model and with the interaction of JD and WT. Because collinearity of predictor variables can yield unstable parameter estimates and inflated standard errors (Quinn and Keough 2002), we verified that JD and WT were not highly correlated $\left(r^{2}<0.6\right)$ before we included them together in a model (Royston and Sauerbrei 2008). We compared models using small-sample-size Akaike information criterion $\left(\mathrm{AIC}_{\mathrm{c}}\right.$ ) to select the "best" model given a candidate set of models and considered models to have equivalent support if $\Delta \mathrm{AIC}_{\mathrm{c}}$ was $<2$ (Burnham and Anderson 2004). We assessed the goodness-of-fit of the global model using a Shapiro-Wilk test $(W>0.90)$.

\section{Results}

Spawning occurred over 30 days at ponds 1-3, beginning on 24 April and ending 23 May (Figure 2).

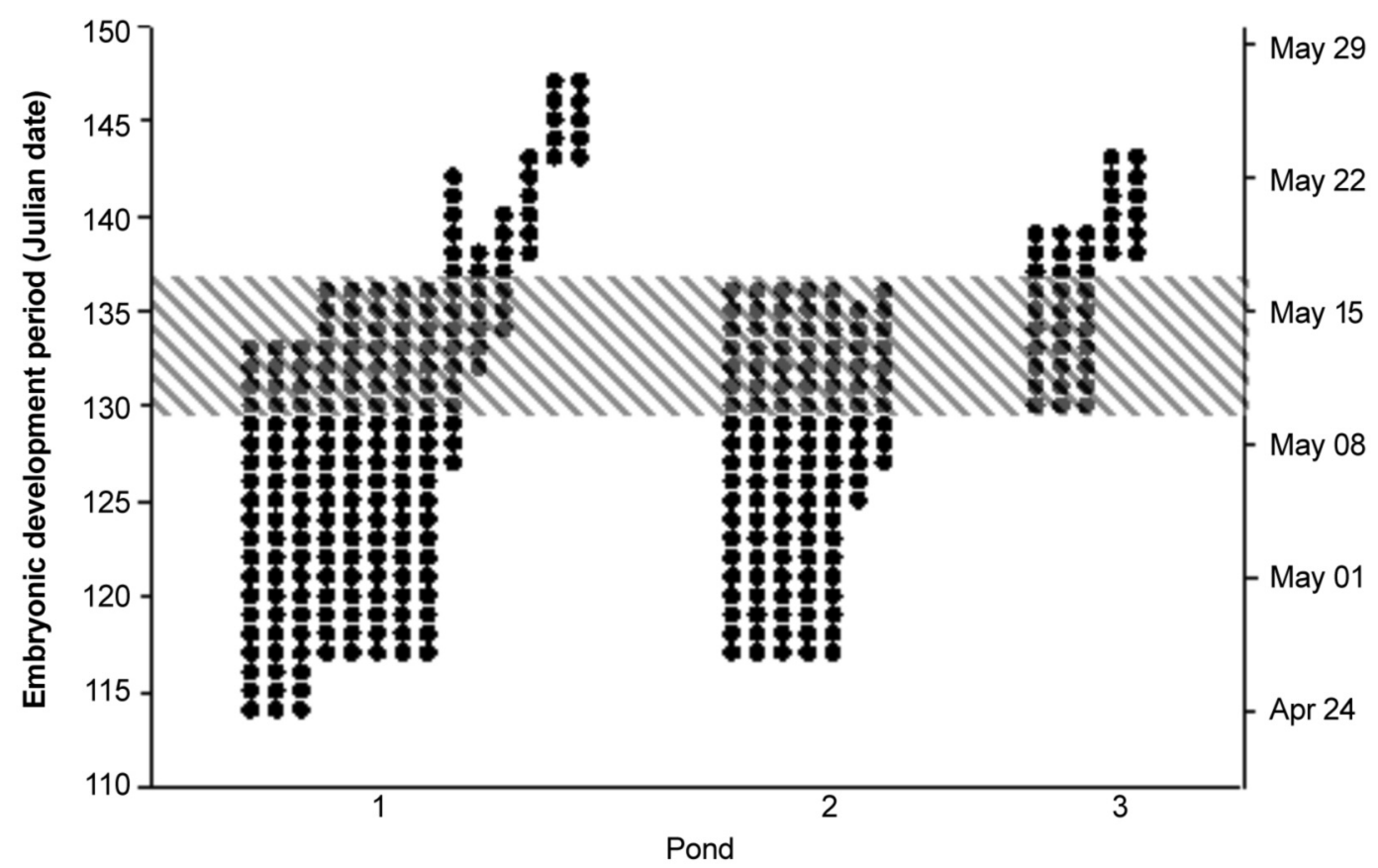

FIGURE 2. Development period for Northern Leopard Frog (Lithobates pipiens) eggs at ponds 1, 2, and 3 in Cypress Hills Interprovincial Park, Alberta (see Figure 1) in April and May 2006. Each column of black circles represents days from spawning to hatching for one of the 26 egg masses observed. The shaded area represents the period during which egg masses were present at all three ponds (10-17 May). 
Although calling and spawning were confirmed at pond 4 , we were unable to monitor egg masses because pollen blanketed more than half of the water surface, affecting our ability to observe them. Hatching occurred at the three remaining ponds over 2 weeks, beginning 14 May with the last eggs hatching on 28 May. We observed a total of 26 egg masses: 14 in pond 1,7 in pond 2 , and 5 in pond 3 . Egg masses were laid within $2-10 \mathrm{~cm}$ of the water surface, typically grouped together in shallow areas within 2-3 m of the shoreline. All monitored egg masses survived to hatching, but we were unable to monitor the proportion of eggs that hatched successfully.

The onset of spawning differed by only 3 days between ponds 1 and 2, but occurred approximately 2 weeks later at pond 3 . The WT near each egg mass on the day of spawning was significantly higher at pond 3 $\left(17.4^{\circ} \mathrm{C} \pm 1.9\right.$ [mean \pm standard error], $r^{2}=0.40, F_{225}=$ 7.62, $P=0.0029$ ) than at ponds 1 and 2 , but did not differ between ponds 1 and $2\left(9.1^{\circ} \mathrm{C} \pm 1.6\right.$ and $9.1^{\circ} \mathrm{C} \pm$
1.1, respectively). At ponds 1 and 2, egg laying first occurred on 24 and 27 April, respectively. Up to five egg masses were laid on the same day in ponds 1 and 2, and the spawning period lasted for almost a month. Egg masses were detected simultaneously at all three ponds during only 1 week, 10-17 May.

At the time eggs were laid WT ranged from $5.7^{\circ} \mathrm{C}$ to $25.8^{\circ} \mathrm{C}$. At pond 1 , WT dipped as low as $3^{\circ} \mathrm{C}$ during egg development (27 April). Time from spawning to hatching ranged from 5 to 20 days and decreased with JD at laying (Figure 3). Eggs deposited at the beginning of the breeding season (24 April) took about four times as long to hatch as the last eggs laid (23 May).

Although we found a strong, negative relation between embryonic development period and JD, the relation between that period and spawning temperature was not as strong (Figure 4). The top model, with 93\% of the $\mathrm{AIC}_{\mathrm{c}}$ weight, was an additive model that included WT and JD (Table 1). No other models were $<2 \Delta \mathrm{AIC}_{\mathrm{c}}$ of the top model.

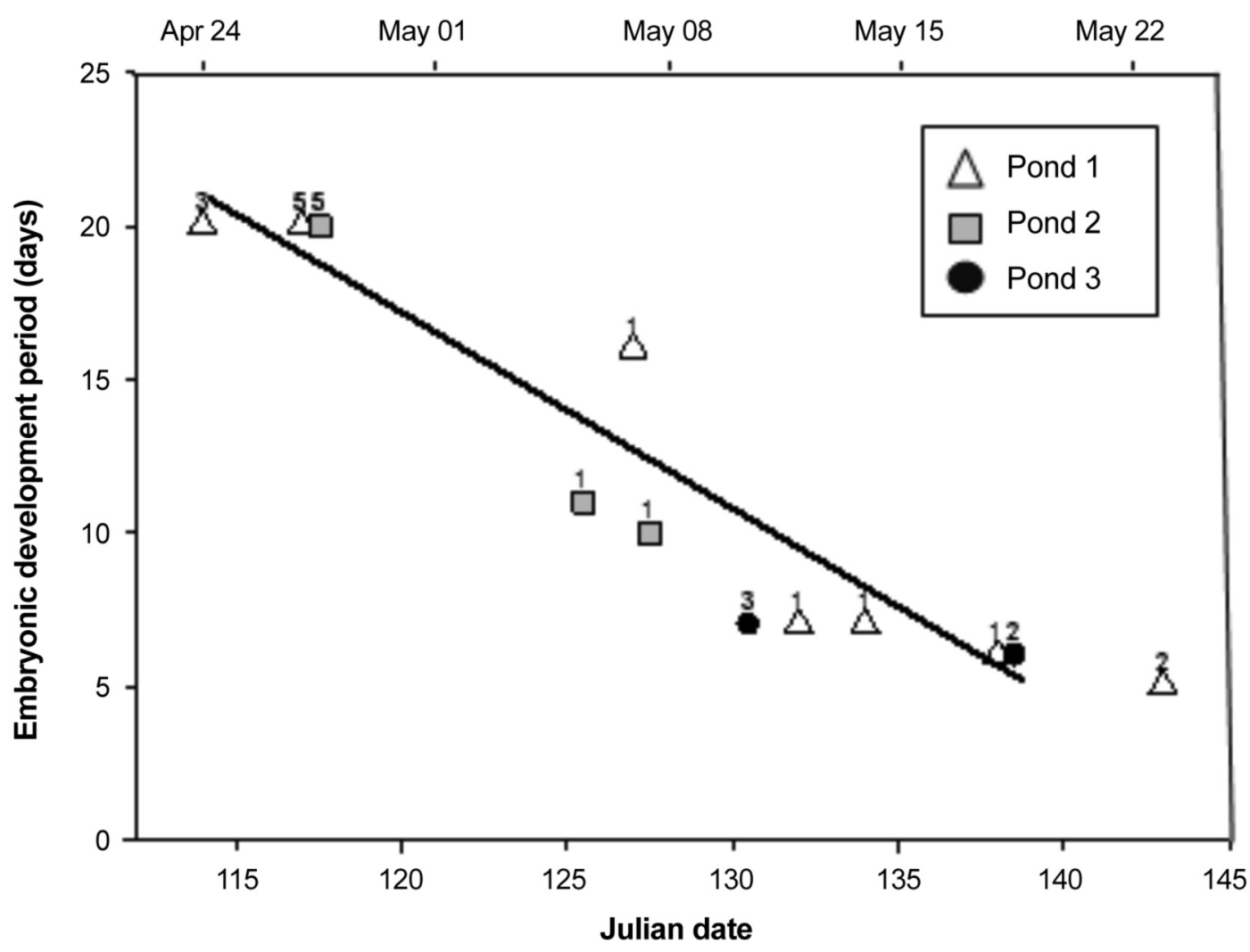

Figure 3. Relation between development period and Julian date (24 April-28 May 2006) for 26 Northern Leopard Frog (Lithobates pipiens) egg masses at ponds 1, 2, and 3 in Cypress Hills Interprovincial Park, Alberta (see Figure 1). The numbers above each symbol represent the number of egg masses laid on that date. Symbols for ponds at which egg masses were laid on the same date have been slightly offset $\left(r^{2}=0.91\right)$. 


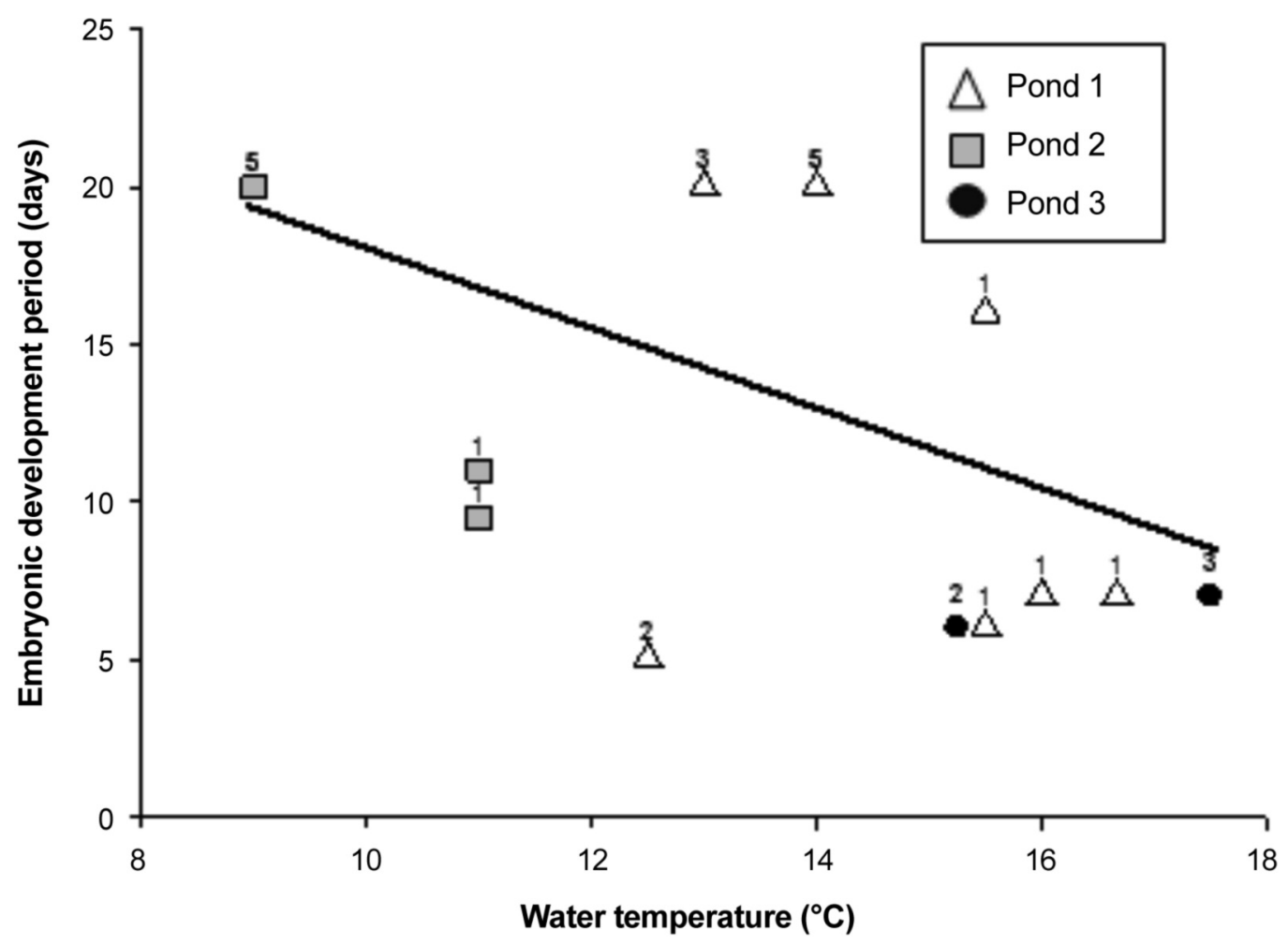

FigURE 4. Relation between development period (24 April-28 May 2006) and spawning temperature for 26 Northern Leopard Frog (Lithobates pipiens) egg masses at ponds 1, 2, and 3 in Cypress Hills Interprovincial Park, Alberta (see Figure 1). Numbers above each symbol represent the number of egg masses laid at that water temperature. Symbols for ponds at which egg masses were laid at the same water temperature have been slightly offset $\left(r^{2}=0.32\right)$.

TABLE 1. Variation in development period of Northern Leopard Frog (Lithobates pipiens) eggs with Julian date (JD) and water temperature (WT). The top model was selected using Akaike information criterion adjusted for small sample size (AIC $\mathrm{C}_{\mathrm{c}}$.

\begin{tabular}{lccccc}
\hline \hline Model & $\mathbf{- 2 L L}$ & $\boldsymbol{K}$ & $\mathbf{A I C}_{\mathbf{c}}$ & $\Delta \mathbf{A I C}_{\mathbf{c}}$ & $\mathbf{A I C}_{\mathbf{c}}$ weight \\
\hline Embryo development period = Pond + JD + WT & 102.59 & 5 & 115.59 & 0.00 & 0.93 \\
Embryo development period = Pond + JD & 111.24 & 4 & 121.14 & 5.55 & 0.06 \\
Embryo development period = Pond + JD +WT + JD*WT & 107.59 & 6 & 124.01 & 8.42 & 0.01 \\
Embryo development period = Pond + WT & 158.07 & 4 & 167.97 & 52.38 & 0.00 \\
Embryo development period = Pond & 164.85 & 3 & 171.95 & 56.36 & 0.00 \\
\hline \hline
\end{tabular}

Note: $-2 \mathrm{LL}$ is $-2 *$ model log-likelihood, $K$ is the number of parameters in the model. $\Delta \mathrm{AIC}_{\mathrm{c}}$ is the difference between the $\mathrm{AIC}_{\mathrm{c}}$ of each model and the top model.

\section{Discussion}

The onset of spawning was not synchronous in our study area and varied by over 3 weeks among our ponds. However, spawning was often synchronous within a pond, with several egg masses laid on a single day. The spawning period was also protracted, lasting up to 30 days at a single breeding pond (pond 1). The duration of the breeding season and the embryonic development period was consistent with other published reports (Merrell 1968; Wells 1977; Russell and Bauer 2000;
Werner et al. 2004). It is interesting to note that, whereas spawning occurred over the course of a month, all eggs hatched within a 2-week period with the first eggs deposited taking almost four times as long to develop as the last eggs laid.

Eggs were laid at WT ranging from $5.7^{\circ} \mathrm{C}$ to $25.8^{\circ} \mathrm{C}$, which is consistent with known egg temperature tolerances for this species (Moore 1939, 1949). However, our minimum spawning temperature was more than $2{ }^{\circ} \mathrm{C}$ colder than that recorded for Northern Leopard 
Frogs in Quebec, which do not spawn at WT below $8^{\circ} \mathrm{C}$ (Gilbert et al. 1994). In addition, WT at one of our ponds dropped to $3^{\circ} \mathrm{C}$ during the development period, which is $2^{\circ} \mathrm{C}$ below the reported threshold for normal embryonic development in this species (Moore 1949). However, the temperature at the centre of an egg mass can be up to $2^{\circ} \mathrm{C}$ warmer than the surrounding water (Hassinger 1970). Although the four affected egg masses survived to hatching, we were unable to evaluate whether the embryos had developed normally.

The top model for embryonic development period included both JD and WT as predictive variables, suggesting that temperature alone is not sufficient to explain differences in development period, which might also be affected by seasonal differences in the region. However, there was no evidence that the effect of temperature on development changed over time (the interactive model was $>8 \Delta \mathrm{AIC}_{\mathrm{c}}$ from the top model). Although it has long been known that the development of Northern Leopard Frogs is temperature dependant (Atlas 1935; Moore 1939), clearly this is not the only factor affecting embryo development period.

\section{Conservation and Management Implications}

Industrial development permits issued by regulators often stipulate that developers determine whether atrisk species, such as the Northern Leopard Frog, are present and establish appropriate mitigation strategies to reduce or eliminate negative impacts of their activities. Often only single surveys are conducted to determine the presence or absence of at-risk amphibians (Kendell 2003*). Our results suggest that inappropriately timed breeding surveys may fail to detect Northern Leopard Frogs and could, thus, limit the ability to develop appropriate strategies to conserve this species.

Even within our small study area, egg masses would have been observed in all three ponds during only 1 week - a narrow timeframe for the completion of effective and comprehensive egg-mass surveys. Because breeding phenology is likely to vary annually, regionally, and locally, we recommend that researchers adjust the timing of their surveys so that they are relevant to each specific site. To identify breeding ponds, multiple breeding surveys separated in time should be conducted. The need to adjust the timing and number of surveys necessary to identify breeding sites is not unique to Northern Leopard Frogs (AESRD 2013*). As such, we recommend that breeding phenology be considered when developing monitoring strategies or industrial mitigation procedures for amphibians elsewhere.

\section{Acknowledgements}

All research was conducted in compliance with University of Calgary Animal Care Protocol BI 2005-39 and the Calgary Zoo BRRC 2005-05 approval number. We are grateful for funding provided by Husky Energy and Cenovus Energy Ltd. We thank Typhenn BrichieriColombi for providing the map of the study area.
Documents Cited (marked * in text)

AESRD (Alberta Environment and Sustainable Resource Development). 2012. Alberta Northern Leopard Frog recovery plan, 2010-2015. AESRD, Edmonton, Alberta. Page 34.

AESRD (Alberta Environment and Sustainable Resource Development). 2013. Sensitive species inventory guidelines. AESRD, Edmonton, Alberta. Page 128.

COSEWIC (Committee on the Status of Endangered Wildlife in Canada). 2009. COSEWIC assessment and update status report on the Northern Leopard Frog Lithobates pipiens, Rocky Mountain population, Western Boreal/Prairie populations, Eastern populations in Canada. COSEWIC, Ottawa, Ontario. 69 pages.

Greenlee, G. M. 1981. Soil survey of Cypress Hills, Alberta, and interpretation for recreational use. Alberta Research Council, Edmonton, Alberta. 77 pages.

Hine, R. L., B. L. Les, and B. F. Hellmich. 1981. Leopard Frog populations and mortality in Wisconsin. Technical Bulletin 122. Wisconsin Department of Natural Resources, Madison, Wisconsin. 39 pages.

Kendell, K. 2002. Survey protocol for the Northern Leopard Frog. Alberta Species at Risk Report. Alberta Sustainable Resource Development, Fish and Wildlife, Edmonton, Alberta. 30 pages.

Kendell, K. 2003. Status of the Northern Leopard Frog (Rana pipiens) in Alberta: update 2003. Alberta Sustainable Resource Development, Fish and Wildlife and Alberta Conservation Association, Edmonton, Alberta. Page 61.

Merrell, D. J. 1977. Life history of the Leopard Frog, Rana pipiens, in Minnesota. Bell Museum of Natural History, Minneapolis, Minnesota. 22 pages.

Roberts, W. 1981. What happened to the leopard frogs? Alberta Naturalist 11: 1-4.

\section{Literature Cited}

Atlas, M. 1935. The effect of temperature on the development of Rana pipiens. Physiological Zoology 8: 290-310.

Burnham, K. P., and D. R. Anderson. 2004. Multimodel inference. Sociological Methods \& Research 33: 261-304.

Dorcas, M. E., S. J. Price, S. C. Walls, and W. J. Barichivich. 2010. Auditory monitoring of anuran populations. Pages 281-298 in Amphibian ecology and conservation: a handbook of techniques. Edited by C. K. Dodd. Oxford Biology, New York, New York.

Dougherty, C. K., D. A. Vaala, and G. R. Smith. 2005. Within-pond oviposition site selection in two spring-breeding amphibians (Ambystoma maculatum and Rana sylvatica). Journal of Freshwater Ecology 20: 781-782.

Fraser, L. 2007. Distribution characteristics of the eggs, tadpoles, and metamorphs of the northern leopard frog (Rana pipiens) and their relation to conservation strategies. MSc Thesis. University of Calgary, Calgary, Alberta.

Gilbert, M., R. Leclair, Jr., and R. Fortin. 1994. Reproduction of the Northern Leopard Frog (Rana pipiens) in floodplain habitat in the Richelieu River, Quebec, Canada. Journal of Herpetology 28: 465-470.

Grant, R. A., E. A. Chadwick, and T. Halliday. 2009. The lunar cycle: a cue for amphibian reproductive phenology? Animal Behaviour 78: 349-357.

Hassinger, D. D. 1970. Notes on the thermal properties of frog eggs. Herpetologica 26: 49-51.

Leonard, W. P., K. R. McAllister, and R. C. Friesz. 1999. Survey and assessment of Northern Leopard Frog (Rana 
pipiens) populations in Washington State. Northwestern Naturalist 80: 51-60.

Merrell, D. J. 1968. A comparison of the estimated size and the "effective size" of breeding populations of the Leopard Frog, Rana pipiens. Evolution 22: 274-283.

Moore, J. A. 1939. Temperature tolerance and rates of development in the eggs of amphibia. Ecology 20: 459-478.

Moore, J. A. 1949. Geographic variation of adaptive characters in Rana pipiens Schreber. Evolution 3: 1-24.

Oseen, K., and R. Wassersug. 2002. Environmental factors influencing calling in sympatric anurans. Oecologia 133: 616-625.

Paton, P. W. C., and R. N. Harris. 2010. Egg mass and nest counts. Pages 143-165 in Amphibian ecology and conservation: a handbook of techniques. Edited by C. K. Dodd. Oxford Biology, New York, New York.

Quinn, G. G. P., and M. J. Keough. 2002. Experimental design and data analysis for biologists. Cambridge University Press, Cambridge, UK.

Reading, C. J. 1998. The effect of winter temperatures on the timing of breeding activity in the Common Toad Bufo bufo. Oecologia 117: 469-475.
Royston, P., and W. Sauerbrei. 2008. Multivariable modelbuilding: a pragmatic approach to regression anaylsis based on fractional polynomials for modelling continuous variables. John Wiley \& Sons, Chichester, West Sussex, UK.

Russell, A. P., and A. M. Bauer. 2000. The amphibians and reptiles of Alberta: a field guide and primer of boreal herpetology. Second edition. University of Calgary Press, Calgary, Alberta.

Ryan, T. J., and G. R. Plague. 2004. Hatching asynchrony, survival, and the fitness of alternative adult morphs in Ambystoma talpoideum. Oecologia 140: 46-51.

Thumm, K., and M. Mahony. 2002. Hatching dynamics and bet-hedging in a temperate frog, Pseudophryne australis (Anura: Myobatrachidae). Amphibia-Reptilia 23: 433-444.

Wells, K. D. 1977. The social behaviour of anuran amphibians. Animal Behaviour 25: 666-693.

Werner, J. K., B. A. Maxell, P. Hendricks, and D. L. Flath. 2004. Amphibians and reptiles of Montana. Mountain Press Publishing Company, Missoula, Montana.

Received 16 September 2013

Accepted 18 November 2013 